\title{
VINCENZO CHIARUGI: LA PAZZIA Y EL REGLAMENTO DE BONIFACIO EN LOS ORÍGENES DE LA PSIQUIATRÍA MODERNA
}

\author{
Norberto Aldo Conti \\ Universidad de Buenos Aires, Universidad del Salvador, Asociación de Psiquiatras Argentinos (APSA) \\ norbertoaldoconti@gmail.com
}

Recibido: 30 de abril de 2015; Aceptado: 1 de septiembre de 2015.

Cómo citar este artículo/Citation: Conti, Norberto Aldo (2015), "Vincenzo Chiarugi: La pazzia y el Reglamento de Bonifacio en los orígenes de la psiquiatría moderna", Asclepio, 67 (2): p105. doi: http://dx.doi.org/10.3989/asclepio.2015.23

RESUMEN: Vincenzo Chiarugi fue director del Hospital de San Bonifacio entre 1788 y 1818. De su experiencia en esa institución para enfermos mentales surgen las dos obras aquí presentadas: 1) Della Pazzia in genere e in spece. Trattato medico-analitico con una centuria di osservazioni, publicado en 1793, en él encontramos un tratamiento de la locura como unidad centrada en el término pazzia, que puede presentar tres aspectos clínicos: melancolía, manía y amencia, que recuerdan bastante a la alienación de Pinel, pero en la pazzia encontramos también la primera referencia a la concepción de enfermedad mental como entidad clínico evolutiva, y 2) Reglamento de Bonifacio, publicado en 1789, en donde expone el régimen institucional y jurídico de la pazzia. Creemos que del análisis de estas dos obras se puede sostener que el pensamiento de Chiarugi está a la base de la conformación de la matriz disciplinar psiquiátrica moderna.

PALABRAS CLAVE: Vicenzo Chiarugi; Pazzia; Reglamento de Bonifacio; Reforma Estatal Toscana.

\section{VINCENZO CHIARUGI: PAZZIA AND THE REGULATION OFBONIFACE IN THE ORIGINS OF MODERN PSYCHIATRY}

ABSTRACT: Vincenzo Chiarugi was director of St Boniface's Hospital between 1788 and 1818.Based on his experiences in this institution he produced two works we now centuria di osservazioni, published in 1793, where madness is treated as a unity centered in the term pazzia, which can present three clinical aspects: melancholy, mania and amencia, that remind us largely of Pinel's alienation. Also, in pazzia we find the firstreference to the conception of mental illness as a clinical and evolutive entity, and 2) The Regulation of Boniface, published in 1789, where he exposes the institutional and legal aspects of pazzia. From the analysis of both works we believe we can state that Chiarugi's conception is at the basis of modern psychiatry disciplinary matrix.

KEY WORDS: Vicenzo Chiarugi; Pazzia; Regulation of Boniface; Tuscan state reform. 


\section{INTRODUCCIÓN}

En el presente trabajo realizaremos una aproximación al pensamiento y la obra de Vicenzo Chiarugi quien impulsó en la Toscana una importante reconversión y reformulación del abordaje médico estatal de la locura en consonancia con los movimientos de reformas en la gestión de la locura ocurridos en Europa entre fines del siglo XVIII y principios del siglo XIX. Es importante destacar que, si bien existe una producción historiográfica en torno a su figura y la gravitación de su obra, desde los trabajos ya clásicos de George Mora en los años cincuenta del pasado siglo (Mora 1954, 1959a, 1959b) y la introducción del mismo autor a la edición inglesa de la obra de Chiarugi (Mora 1987), pasando por los autores italianos que se han ocupado de él (Scapini, 1966; Stroppiana, 1976; Cabras, Campanini, Lippi, 1993; Pallanti, 1996; Lippi, 2008), y otros que abordan aspectos específicos de su obra (Lantéri-laura, 1994; Charland, 2014), o el análisis de su controvertida relación con Pinel (Grange, 1963; Gerard, 1998), su presencia sigue siendo hoy marginal en la mirada de conjunto que se tiene acerca del surgimiento de la psiquiatría moderna en Europa como oportunamente lo ha destacado Rafael Huertas (1999; 2005).

\section{VINCENZO CHIARUGI, EL HOMBRE}

Vincenzo Chiarugi nació en Empoli, localidad Toscana distante treinta kilómetros de Florencia, el 20 de febrero de 1759. Su madre, Margherita Conti, murió en el parto y su padre, Antonio Gregorio Chiarugi, fue el primer médico en una familia de comerciantes de la ciudad de Prato cuyos orígenes se remontaban al siglo XIV. Vicenzo realizó sus primeros estudios en Empoli y luego estudió en Pisa, en el Collegio Ferdinando, donde se doctoró en Filosofía y Medicina el 19 de mayo de 1779. Realizó luego el año de práctica obligatoria en el Hospital de Santa María Nueva, en Florencia, obteniendo el 23 de junio de 1780 la licencia para ejercer la profesión. (Maggini, Pintus, 2002; Stagnaro, 2014).

En 1782 fue nombrado médico asistente, encargado de la guardia, en el Hospital de Santa María Nueva y en 1785 pasó a desempeñarse como médico superintendente del Servicio de Hombres del Hospital de Santa Dorotea en donde comenzó a tomar contacto con la locura y, durante esa experiencia, prohibió el uso de cadenas en la contención de los pacientes excitados.

En ese mismo año de 1785 nació su primogénita Margharita, fruto del matrimonio de Vicenzo con
Migliorotta Ricci, instalándose entonces en la Via Pérgola de Florencia, cercana al hospital. La familia se completará con la llegada de los otros hijos: Antonio (1786), José (1787), Lorenzo (1789), Luisa (1793), Teresa (1797) y Eleonora (1801); en esos años la familia se mudará varias veces para, finalmente, afincarse en una gran casa en la Vía larga de Florencia, cercana también a los hospitales en donde se desempeñaba Chiarugi (Lippi, 1992; Stagnaro, 2014,)

Durante el año 1786 participó de la renovación del Hospital de Bonifacio bajo las órdenes del Gran Duque Pedro Leopoldo; este proceso, al que Chiarugi adhiere fervientemente, se inscribe en el contexto de renovación de la legislación sobre la locura en Toscana iniciado con la promulgación de la primera ley sobre los enfermos mentales en Europa (Legge sui pazzi) de 1774. Toda su labor clínica y sanitaria estará orientada en esta dirección y se plasmará en sus obras como veremos más adelante. En 1788 fue nombrado Jefe de Servicio del nuevo hospital y poco después Director, cargo que ocupó hasta 1818. (Maggini, Pintus, 2002, Postel y Quetel, 2000, Guarnieri, 1991).

En 1793 publicó el primer tomo de su obra más importante: Della Pazzia in genere, e in specie. Trattato médico analítico con una Centuria di Osservazioni, y en 1794 aparecieron los dos tomos siguientes publicados en un solo volumen. La obra, que tuvo una gran acogida, fue traducida al alemán en 1796 y presentó una segunda edición, profundamente modificada, en 1808. (Stagnaro, 2014; Morel, 1996; Cabras, CampaniIli, Lippi, 1993; Riefolo, 1991; Berti Bock, 1971).

En cuanto a su vida académica Chiarugi fue Profesor de Enfermedades Cutáneas y Mentales en la Escuela de Medicina de Florencia en 1802 y también Profesor Honorario de la Universidad de Pisa en 1810.

También tuvo importantes actuaciones como clínico y sanitarista. En 1804, durante una epidemia de fiebre que azotó la Toscana, se le encargó ocuparse de ella y organizó las operaciones sanitarias necesarias para su resolución, volviendo a cumplir esta función durante una epidemia de tifus que aquejó a Florencia en 1817 (Stagnaro, 2014).

En 1806 pasó a ser miembro de la Sociedad Colombaria $^{1}$, de la Academia Florentina ${ }^{2}$ y de la Sociedad Italiana de Ciencias, Artes y Literatura.

En 1807 ingresó al Colegio Médico Florentino, esta institución, que estaba organizada en tres secciones: Medicina, Cirugía y Farmacéutica, le permitió relacionarse con las autoridades científicas de la época. 
En 1819 accedió al cargo de Superintendente de la Enfermería del Hospital de Santa María Nueva y se le encomendó la enseñanza de Fisiología, Patología y Terapéutica para el curso de especialización, en ese contexto confeccionó el reglamento de los exámenes que debían aprobarse para acceder a las matrículas de médico, cirujano y farmacéutico.

El reconocimiento a sus ideas reformistas hizo que fuera llamado como consultor durante la reforma del Instituto Hospitalario de San Servolo, en Venecia, transformado en el manicomio mixto central de Veneto, Dalmacia y Tirol (Stagnaro, 2014).

La obra escrita y publicada por Chiarugi, más allá de la Pazzia que aquí nos ocupa, se completa con: Lettere sopra un caso di mao venereo (Firenze, 1783), Saggio teorico-pratico sulle malattie cutanee sordide osservate nel Regio Spedale di Bonifazio a Firenze (Firenze, 1799), Istoria delle malattie afrodisiache e di quelle malattie ostinate e non guarite dall'arte medico-chirurgica venute nel $R$. ospedale di Bonifazio negli anni 1802 e 1203 (Firenze, 1804), La fisica dell'oumo, ossia corso completo di medicina ad uso degli ufficiali di sanitá (Firenze, 1811), Saggio di ricerche sulla pellagra (Firenze, 1814) y Principi elementari di Medicina Teorica (Firenze, 1820) (Stagnaro, 2014).

Fallece, a causa de una afección pulmonar ligada a la gota, y es enterrado en Florencia el 20 de diciembre 1820. Finalmente, en 1929 , es trasladado a la Iglesia de Santo Stefano degli Agostiniani, en su Empoli natal. (Pallanti, 1996; Maggini, Pintus, 2002; Shorter, 2005).

\section{LA REFORMA ESTATAL DEL GRAN DUCADO DE TOS- CANA (1770-1790): EL RÉGIMEN DE LA LOCURA Y LAS INSTITUCIONES}

En 1745 Francisco Esteban de Lorena alcanza el trono del Sacro Imperio Romano-Germánico y toma el nombre de Francisco I, en 1747 nace en Viena su segundo hijo, Pedro Leopoldo, quien a la muerte de su padre, en 1765, hereda el título de Gran Duque de Toscana, mientras que su hermano mayor, José II de Austria, recibía la corona del Sacro Imperio.

Pedro Leopoldo llevó adelante la reforma administrativa y jurídica del Gran Ducado y, a la muerte de su hermano José II, dejó el Gran Ducado de Toscana y fue coronado emperador del Sacro Imperio Romano-Germánico y rey de Alemania e Italia con el nombre de Leopoldo II el 9 de octubre de 1790. En poco menos de un año sumó también a su poder político los reinos de Hungría, Croacia y Bohemia.
Leopoldo II gobernó durante el momento más dificultoso para las monarquías ilustradas y reformistas europeas: la ocasión y los efectos de la Revolución Francesa; desplegó una intensa actividad política y diplomática para mantener la paz en sus reinos pero su gobierno no alcanzó los dos años muriendo el 1 de marzo de 1792.

En 1790, al asumir la corona del Sacro Imperio, Leopoldo II entregó el Gran Ducado de Toscana a su hijo Fernando III, quien lo gobernó hasta 1801 cuando Napoleón lo obligó a entregarlo a los Borbones de Parma formándose así el reino de Etruria compuesto por los ducados de Toscana y Parma; en 1807 desaparece el reino de Etruria y la Toscana pasa a ser gobernada por Elisa Bonaparte, en nombre del imperio francés. A la caída de Napoleón, en 1814, Fernando III recupera el Gran Ducado y lo gobierna hasta su muerte en $1824 .^{3}$

En líneas generales las reformas apuntaron a dar un gran impulso a la economía, la agricultura, el comercio y la industria, a democratizar la administración y el manejo fiscal, eliminando privilegios y haciendo público el presupuesto estatal. También a mejorar la calidad de vida de la población a través del desarrollo de las obras públicas y la modernización de servicios. Con este propósito también se llevó adelante la reforma del Código Penal en 1786 (que abolió el delito de lesa majestad, la confiscación de bienes, el interrogatorio bajo tortura y la pena capital) y la reforma en la asistencia de los locos iniciada con la ley sobre locos de 1774 y que culmina con el Reglamento del Nuevo Hospital de Santa María Nueva y de Bonifacio, orientado por Chiarugi y promulgado en $1789^{4}$ (Stagnaro, 2014).

Antes del inicio de la reforma de la asistencia de los locos existían en Florencia varias instituciones que albergaban enfermos mentales, aunque para la época que nos ocupa solo mantenían una función custodial y no terapéutica, la reforma modificó el régimen de asistencia de los locos e indicó el traslado de todos ellos al Hospital de Bonifacio. Haremos una breve referencia ellas y luego nos ocuparemos del Hospital de Bonifacio y su Reglamento.

El Hospital de Santa Dorotea fue inaugurado en 1643 con la misión de albergar a los locos que hasta ese entonces eran encerrados de por vida en la cárcel de Florencia (Stinche), siendo un lugar pequeño, en poco tiempo tuvo problemas de hacinamiento y en 1753 los pacientes fueron trasladados a un antiguo hospital cerrado desde 1541, el Hospital de San Niccoló del Ceppo; finalmente y, en el movimiento de la 
reforma, Pedro Leopoldo decretó, en 1785, que todos los locos allí encerrados debían pasar al Hospital de Bonifacio, medida que se efectivizó en 1788.

El Hospital de Santa María Nueva fue fundado en 1288 y representó un ejemplo temprano y eficaz de atención de la salud en Europa, durante el gobierno de los Médici fue refaccionado y optimizó sus funciones. En 1688 se construyó una pequeña unidad hospitalaria que se destinó como Pazziaria o Casa de Locos recibiendo solo varones, y, a partir de 1729, también se recibieron mujeres. Al igual que en el caso anterior los pacientes de la Pazziaria de Santa María Nueva fueron trasladados al Hospital de Bonifacio durante la reforma. (Tombaccini, Lippi, Lelli, Rossi, 2008).

El Hospital de Bonifacio tiene como antecedente la creación en 1377 del Ospedale San Giovanni Battista, construido gracias al aporte de Bonifacio Lupi de Parma (1316-1390) influyente político toscano. Esta institución, que estaba destinada a albergar pobres e indigentes, se encontraba ubicada entre el convento de Santa María del Querceto y el Ospedale dei Broccardi, en 1436 ambos edificios fueron anexados al hospital y este comenzó a llamarse Hospital de Bonifacio en honor de su fundador. En 1736 el Bonifacio devino exclusivamente depósito de inválidos trasladándose los enfermos a otros hospitales. Finalmente durante el gobierno de Pedro Leopoldo esta institución fue totalmente refaccionada y ampliada transformándose en una unidad sanitaria ejemplar en el contexto de la reforma de la asistencia pública. Se la subdividió en cinco servicios destinados a locos, incurables, enfermos de la piel, soldados e inválidos. (Zandri, Acidini Luchinat, Francolini, 1989).

A partir del 19 de mayo de 1788 el Hospital Real de Bonifacio se ocupó de la asistencia de todos los enfermos mentales de Florencia bajo la dirección de Vincenzo Chiarugi contando, a mediados de ese año, con 569 pacientes internados.

Chiarugi recuerda, con estas palabras, ese momento fundacional de la reforma del régimen de la locura:

Cuando el deplorable estado del antiguo Hospital de Santa Dorotea, destinado a la custodia de los locos, le planteó a los poderes públicos la necesidad de procurar a estos miserables enfermos un mejor destino, fue emprendida, y en poco tiempo concluida, la construcción de una institución provista de todas las comodidades necesarias para favorecer su curación, o al menos para hacer su vida menos incómoda y atormentada.
Con el marco de una construcción dotada de una elegancia y una magnificencia verdaderamente palaciegas, se estableció un método en el mantenimiento y en la asistencia, tan preciso, seguro y ordenado, que ha recibido la admiración de los más cultos viajeros, de respetables personajes, y también de algunos monarcas, que han honrado el nuevo hospital con su visita, el cual recibió el nombre de Bonifacio, que heredó del antiguo asilo anexo de pobres e inválidos (Chiarugi, 1793, t l, p. 2).

\section{Reglamento del Nuevo Hospital de Santa María Nue- va y de Bonifacio (1789)}

Se trata de un documento de 416 páginas en el cual se desarrolla la historia, el inventario de equipamiento y bienes de ambas instituciones, el reglamento del personal, la organización de la Escuela de Medicina, el régimen institucional de abordaje y vida cotidiana de los locos hospitalizados y el régimen jurídico de su internación ${ }^{5}$. Analizaremos aqueIlos aspectos más destacables en el contexto del presente trabajo.

\section{La escuela de Medicina}

El objetivo de la Escuela del Hospital de Santa María Nueva será proporcionar al médico clínico la instrucción teórico-práctica necesaria que no puede ser brindada habitualmente en una Universidad Pública, y formar un hábil cirujano tanto como un experto e iluminado especialista. Con tal propósito se contará con ocho cátedras que se ocuparán de los siguientes temas: Medicina Práctica, Anatomía, Instituciones Quirúrgicas, Casos Prácticos de Cirugía y de Operaciones Quirúrgicas en Cadáveres, Obstetricia, Botánica y Materia Médica, Química y Farmacia (Reglamento, 2da. Sección, citado en Stagnaro, 2014, pp.28).

\section{Régimen de abordaje del paciente}

Fue diseñada en el Santa María Nueva una nueva y detallada planilla de datos que debe fijarse en la cama del correspondiente enfermo indicando su nombre y apellido, lugar de origen, estado civil, tratamiento, nombre y apellido del médico tratante, fecha de admisión en el hospital y del comienzo de la enfermedad, diagnóstico y tratamiento de la misma, las órdenes y observaciones anotadas cotidianamente y el resultado del tratamiento hasta la completa curación o el detalle de la disección del cadáver en caso de muerte (Reglamento, Introducción, citado en Stagnaro, 2014, pp. 26). 


\section{Régimen institucional}

Los principios del régimen institucional están centrados en el rechazo al uso de la violencia física, el confinamiento, el hacinamiento y la desprotección de los locos. Para ello se dispone de una organización del espacio con amplias habitaciones y baños marmóreos higiénicos, con agua corriente en todo el edificio y aire puro y ventilado. Posee también jardines y galerías abiertas con paseos arbolados para el descanso y esparcimiento de los pacientes con la expresa prohibición de visitas de curiosos. La alimentación es sana y abundante (180 gr. de carne por día) y los internos están separados por sexo y grupos clínicos para su mejor asistencia y cuidado. Se programan diferentes actividades, en cada caso según las indicaciones médicas, entre las cuales se cuentan las prácticas religiosas, paseos, excursiones en inclusive algunas tareas laborales. La contención física, tan frecuente en esa época, solo está autorizada en situaciones extremas con ataduras de tela de algodón y brazaletes de cuero.

\section{Régimen jurídico de internación}

Los enfermos que se enviaban al Manicomio debían ir acompañados de una orden del Juez y del Alcalde, y de un certificado médico en el que se señalasen las causas de la enfermedad, el origen inicial, los síntomas y los principales aspectos relacionados. Así, el Gobierno, el Ayuntamiento y los médicos se declaraban garantes, frente a la sociedad, de lo justo y conveniente del secuestro... ${ }^{6}$.

Trattato Della Pazzia: psicopatología, nosografía y clínica a fines del siglo XVIII en Florencia

En esta obra Chiarugi utiliza el término pazzia, que remite a locura en el uso cotidiano de la lengua toscana, con un sentido técnico para hacer referencia a enfermedad mental en general y muy cercano al término alienación, utilizado poco después por Pinel con los mismos fines. Define entonces a la pazzia como un delirio crónico permanente, sin fiebre y en estado de vigilia, delimita así a la locura, en sentido médico, como una enfermedad unitaria y primaria diferenciada de los cuadros tóxicos y febriles y de las conductas oníricas. Pero esta enfermedad mental se expresa en tres presentaciones clínicas diferentes y susceptibles de pasar de una a otra durante la evolución de un mismo paciente, se trata de la melancolía, la manía y la amencia. Esta concepción de unidad de la psicosis con diferentes presentaciones clínicas remite al concepto de monopsicosis o psicosis única, de la cual Chiarugi es considerado el iniciador (Huertas, 1999), y que será seguido después, en el siglo XIX, por los trabajos de Guislain (1797-1860), Zeller (1804-1877), Neumann (1814-1884) y, principalmente, Griesinger (18171868), en su tratado de $1845^{7}$.

Definida la pazzia como un delirio, lo primero que hace Chiarugi es delimitar el concepto de delirio y presentar sus géneros y características. Para ello comienza con una declaración de principios: el hombre está constituido por res cógitans y res extensa, alma y cuerpo:

esa sustancia espiritual, inextensible, inalterable e indestructible que, junto con la otra parte, el cuerpo, material y caduca, concurre a la formación del hombre [7] ${ }^{8}$.

Y puesto que la unión de estas dos sustancias es tan estrecha que una apenas puede actuar sin que la otra sea afectada, es en consecuencia necesaria que, afectadas las funciones de una, también se deba alterar la economía de la otra [8].

La razón es un atributo del alma y organiza la relación del hombre con el entorno y consigo mismo en condiciones normales, para ello concurren el intelecto y la voluntad, en tanto cualidades del alma, y la imaginación y la memoria en tanto sus operaciones. Mediante la razón el hombre compara las ideas y emite juicios, los cuales articulados constituyen el razonamiento. Pero, a veces, sostiene Chiarugi, se produce una falla en este proceso debido a una alteración de la voluntad y a un error del juicio: "Este estado del Hombre, o sea, esta alteración del juicio, y en consecuencia del raciocinio, es lo que se llama Delirio, un estado que muestra como puede ser llevada la naturaleza humana al extremo más humillante..." [15].

Tenemos entonces que el delirio es producido por una alteración en las cualidades y operaciones del alma y por otro lado es el fundamento de la pazzia la cual, en tanto enfermedad, interesa a la materia corporal, se pregunta entonces Chiarugi, ¿Dónde estará la sede de los delirios?, ¿En dónde se dará esta interacción recíproca entre el alma y la materia?, la respuesta lo lleva a desarrollar su concepto de Sensorio Común:

... la máquina que sirve para el ejercicio de las facultades respectivas es el Sistema Nervioso, del cual el cerebro es el centro y la fuente principal y única. Esta víscera se compone de dos sustancias: Cortical y Medular, distintas y limitadas entre ellas" [22].

... en el Cerebro radica un lugar de encuentro de estos orígenes nerviosos (cortical y medular), donde 
el Alma está probablemente presente, más que en las otras partes del Sistema Nervioso, y en la que debe razonablemente suponerse que se encuentra la sede de la comunicación de los movimientos nerviosos. Este lugar es precisamente ese órgano... al que le he dado el nombre de Sensorio Común" [23].

... el Sensorio Común reside donde termina la Sustancia Cortical y principia la Medular. Allí es donde parece ser que el Alma tiene la relación más inmediata con el Cuerpo, y donde ella desarrolla y hace actuar sus facultades, como una Reina en su propio palacio [24]

De esta manera Chiarugi ubica en la materialidad del cerebro el origen de la locura en tanto enfermedad sin afectar la inmutabilidad del alma y considerándola sustrato de la operaciones mentales que están a la base del trastorno, una digna salida a las exigencias de un racionalismo más cercano a la Escolástica que a la llustración.

Definido así el Sensorio Común puede volver sobre la pazzia para conceptualizarla de la siguiente manera: "Las Locuras son errores del Juicio y del Razonamiento procedentes de una afección idiopática del Sensorio Común, sin acompañamiento de fiebre primitiva o de afección comatosa" [45].

Y luego precisa el estado de locura con un párrafo de llamativa similitud estructural con aquel otro famoso en el cual Esquirol, en $1838^{\circ}$, describe el estar alucinado:

Podrá decirse loco con justicia al infeliz individuo de la especie humana que sin tener enfermedad febril o lesiones de los sentidos externos y fuera de las circunstancias del sueño, embriaguez y otras causas similares, demuestra tener sensaciones que no tienen otros en la misma situación, actúa o razona de una manera contraria a las sensaciones de las que tenemos razones para suponer que debería tener, y por último, está persuadido de un error, en sí mismo fácil de reconocer, que antes no lo habría engañado, y sin que la fuerza de la razón y el testimonio de los sentidos pueda convencerlo.

Esta pazzia o locura, causada por una alteración idiopática del Sensorio Común se expresa en esas tres formas fenoménicas ya mencionadas, melancolía, manía y amencia de las cuales quisiéramos dar ahora algunas precisiones nosográficas y clínicas.

En primer lugar, ¿A qué tipo de trastornos se refiere Chiarugi cuando utiliza estos términos?, veamos como los delimita:

\section{Melancolía}

... Una forma de raciocinio erróneo parcial, dependiente de un falso principio puesto $y$, al mismo tiempo, adoptado como base del juicio, que actúa como un centro al que tienden todos los discursos correctamente conducidos y procedentes en sí, pero erróneos, porque dicho principio, del cual provienen, no se corresponde con la verdad. El resto de las acciones y los razonamientos que no se relacionan con este principio corresponden a las nociones de la razón normal y a los testimonios de los sentidos [48].

\section{Manía}

... Una desconexión general de ideas con respecto al presente, el pasado y el futuro, y sin distinción de las relaciones; acompañada por una rápida transición que va de una serie de pensamientos a otra, absolutamente ajena, una excitación y audacia inusuales, y por el impulso y la violencia en todos los juicios, que lo llevan a tener un lenguaje y un comportamiento muy coléricos y furiosos [48].

Amencia

... Una irregularidad o imperfección general, o casi general, en las operaciones del intelecto y de la voluntad. Usualmente sin emociones precisas, ya sean de tristeza o de cólera, y con debilitamiento e imbecilidad en todas sus acciones [48].

$Y$ en el siguiente parágrafo nos brinda, en un intento de alcanzar la mayor claridad conceptual, una definición desprovista de ropaje clínico que apunta a la esencia y diferencia entre cada una:

La Melancolía es una Locura parcial limitada siempre a uno o unos pocos temas relacionados entre sí [49].

La Manía es una Locura general acompañada de la audacia y el furor en las acciones voluntarias [49] ${ }^{10}$.

"La Amencia es una Locura general o casi general, con alteraciones en las capacidades intelectuales y volitivas, pero habitualmente sin emociones [49].

Pero, luego de estas claras definiciones, afirma que:

... los síntomas de estos tres tipos de Locura a menudo se mezclan, se suceden y se reproducen recíprocamente [...] usualmente no pueden ser aislados individualmente con exquisitez y precisión en un individuo... [50].

Esto quizás podría hacernos creer que [...] cada uno constituye más bien una presentación diferente de un fenómeno común a todos ellos [50]. 
Encuadra así las tres manifestaciones clínicas de la pazzia en el ordenamiento conceptual de la Psicosis Única, como lo habíamos referido anteriormente. Respecto a la sucesión cronológica de las tres variedades, ya esbozada en el parágrafo anterior, dice: “... Puedo asegurar que he visto, en la mayoría de los casos, que cada grado de Locura principia con la Melancolía, la que fácilmente pasa a Manía; al finalizar una, la otra se reproduce y finalmente de estas dos fuentes se origina la Amencia" [53].

Podemos reconocer aquí que Chiarugi, al igual que otros autores del siglo XVIII ${ }^{11}$, observa en su clínica, cotidianamente, el pasaje de melancolía a manía lo cual es interpretado, en el entramado de su psicopatología, siguiendo la lógica secuencial de la Psicosis Única.

Pero esta novedad, la de la importancia de lo diacrónico en la tipificación de la locura, no solo la encontramos en la sucesión de las variedades propuestas sino también en el encadenamiento temporal de los síntomas y de las circunstancias que precedieron a la aparición de los mismos, como lo describe en el caso de las manías: "... Solamente combinando una observación diligente sobre el estado actual con conocimiento adecuado de las circunstancias que precedieron la Manía seremos capaces de decidir con certeza sobre la verdadera especie de enfermedad presente"[572].

Utilizando esta modalidad de observación centrada en la diacronía organiza el diagnóstico diferencial de los diferentes tipos de manía articulando los antecedentes con la clínica e incluso, en algunos casos, pronosticando la evolución esperable. Este abordaje constituye un antecedente del concepto de entidad clínico-evolutiva ${ }^{12}$ que tradicionalmente no se verifica antes de los trabajos de Jean Pierre Falret (1794-1870), de $1854^{13}$ y Karl Khalbaum (1828-1899), de $1874 .{ }^{14}$

Analizaremos ahora las diferentes variedades de estas tres presentaciones (melancolía, manía y amencia) para luego poder tener una mirada de conjunto de su nosografía.

Al abordar el análisis de la melancolía (Cap. I, Libro I, Segunda Parte: Especies de la Locura), Chiarugi afirma que:

... la Melancolía radica... en esencia, (en que) la mente divaga sobre objetos reales y verdaderos, pero razona sobre ellos de una forma anormal... [397].
... el Hombre situado en esta convicción... se ve ubicado en tres estados diferentes relacionados con sus propias pasiones. $\mathrm{O}$ bien son pasiones tristes y los individuos... se ven oprimidos por la tristeza y el temor, o bien se sienten estimulados o capaces de atemperarse entre sí, en cuyo caso sienten alegría o tranquilidad; o bien, finalmente, son mixtas; particularmente pertenecientes al odio, y es aquí donde aparecen las acciones de ira y de audacia desmedida [398].

... Ilamaré Melancolías Verdaderas a todas aquellas acompañadas por la tristeza y el miedo [400].

... he dado el nombre de Melancolías Espurias a todas aquellas acompañadas por la alegría o la tranquilidad [401].

... he llamado Melancolías Furiosas a todas aquellas acompañadas por una audacia y una furia parcial [402].

Vemos entonces que al utilizar el término verdaderas para las presentaciones que cursan con tristeza y miedo respeta la clásica concepción hipocrática acerca de la melancolía pero al agregar las otras dos formas se impone el ordenamiento de Willis en locuras generales o parciales, quedando las melancolías consideradas como parciales, aclaración con la que cierra el capítulo: “... Pero en cada uno de estos casos, la mente está fija en un solo objeto, o en varios relacionados... coincidiendo todos en el carácter principal." [404]

En el libro II, dedicado al análisis de la manía, realiza una descripción minuciosamente rica de los síntomas que se suceden en un orden determinado y que le permite proponer para ellos tres etapas: Acceso, Estado y Remisión operando con un criterio clínico evolutivo, como ya habíamos observado:

La audacia y la furia que acompañan el delirio general de la Manía forman el signo patognomónico de esta enfermedad, pero como hay tantos fenómenos que se unen y tantas son las modificaciones que sufre en su curso, creo conveniente dividir la enfermedad en tres etapas: de Acceso, de Estado y de Remisión, cada uno caracterizado por síntomas diferentes [556].

Se ocupa también aquí, al enumerar las causas de la manía, de proponer un ordenamiento o clasificación etiológica:

I. Manía Mental: la que se produce por la acción inmediata del Alma. II. Manía Reactiva: la que surge del debilitamiento de la actividad de la potencia nerviosa. III. Manía Pletórica: la que es producida por la plétora de sangre en el sistema vascular. IV. Manía Inmediata: 
la ocasionada por un estímulo actual, que produce inmediatamente en el Cerebro el depósito de materias morbosas. V. Manía consensual: la que es originada por la afección de cualquier parte del organismo que tenga relación nerviosa con el Sensorio Común [554].

Al inicio de la presentación de cada una de estas cinco formas explicita más claramente su adhesión al criterio clínico evolutivo ya que, luego de afirmar la necesidad del conocimiento de las circunstancias que precedieron a los síntomas para decidir con certeza, afirma:

Pero como no siempre es posible tener informaciones preliminares como para formar una base sobre la cual razonar, será necesario tener en cuenta solamente los signos particulares, con el fin de establecer una probabilidad conjetural suficiente, con la que generalmente es necesario que se contenten los médicos sinceros y de mente abierta [573].

Estaría entonces claro, para Chiarugi, que el diagnóstico de certeza es posible de alcanzar si contamos con información suficiente sobre las circunstancias que precedieron la aparición de de un determinado cuadro, aunque contraste este principio con la realidad de la ausencia de información preliminar y por este motivo reduzca la veracidad del diagnóstico al nivel conjetural.

Finalmente el Libro III está dedicado a la Amencia la cual, a diferencia de la Melancolía y la Manía, es presentada como una afección irreversible y esto se debe a que: “... en la Amencia, la alteración del Sensorio Común de la cual dependen dichos razonamientos erróneos y los demás síntomas es tan crónica que a menudo es incurable"[740].

A la hora de precisar el origen del término y su configuración clínica afirma:

... un hombre que se deja llevar por su instinto, por el hábito, por el apetito y no por la Razón, y que no muestra los efectos producidos en él por la potencia razonadora, o sea por la Mente, sufrirá una carencia de su condición humana; y por eso se le dará el nombre de Amente, o sea, sin Mente [739].

... los individuos afectados advierten poco o nada lo que les ocurre y no son movidos por las pasiones que, en iguales circunstancias, conmueven a los hombres normales" [738].

Al analizar el conjunto de las Amencias reconoce en ellas dos variedades: aquellas en las cuales, a pesar de la marcada imperfección de las operaciones mentales, aún se observa una cierta movilidad y sucesión de ideas a las que llama Amencias Activas y aquellas en las que se observa una marcada lentitud en dichas operaciones a las que llama Amencias Defectivas. Considera variedades de la primera Fatuidad, Necedad e Imbecilidad y variedades de la segunda Olvido y Estupidez.

\section{LA LOCURA, SUS GÉNEROS Y ESPECIES (1793)}

\section{MELANCOLÍA}

MELANCOLÍA VERDADERA

MELANCOLÍA ESPÚRIA

MELANCOLÍA FURIOSA

\section{MANÍA}

MANÍA MENTAL

MANÍA REACTIVA

MANÍA PLETÓRICA

MANÍA INMEDIATA

MANÍA CONSENSUAL

\section{AMENCIA}

AMENCIAS ACTIVAS: Fatuidad, Necedad, Imbecilidad

AMENCIAS DEFECTIVAS: Olvido, Estupidez

\section{CONCLUSIONES}

La reforma toscana del régimen de la locura, instituida por Chiarugi en Florencia, antecede en por lo menos una década a la llevada a cabo en Francia y resulta exitosa al menos hasta después de 1839 (Huertas y Del Cura, 2004), extendiéndose por más de cincuenta años.

El abordaje de la locura como problema médico realizado por Chiarugi en su Pazzia presenta una serie de conceptos que serán luego trabajados por diferentes autores a lo largo del siglo XIX, a saber, la unidad de la locura y la pluralidad de variedades (Alienación Mental), la secuencia regular y cronológica de aparición de las variedades en un individuo (psicosis única) y la relación antecedentes - clínica actual- pronóstico, núcleo de la concepción de entidades clínico-evolutivas (enfermedades mentales).

Vemos en definitiva que la obra institucional y teórica de Chiarugi está en la base de las conceptualizaciones que la floreciente psiquiatría del siglo XIX desarrolló tanto en Francia como en Alemania e Inglaterra. 
Si entre la una y las otras se pueden inferir relaciones causales o se trata solo de un efecto de época es un interrogante que estimula el renovado interés en la investigación de este período fundacional de la psiquiatría, como muy bien lo expresan Juan Carlos Stagnaro y Rafael Huertas en este Dossier.

\section{NOTAS}

1 Sociedad Fundada en Florencia por el caballero Pazzi en 1735, congregaba a los sabios de la ciudad que se reunían en la parte más alta del Palacio Pazzi, de allí su nombre de Sociedad Colombaria.

2 También conocida como Academia Platónica Florentina, fue fundada por Cosme de Médicis en 1459 y tuvo entre sus miembros a Marsilio Ficino, Pico della Mirándola, Savonarola y el Cardenal Bessarion.

3 Dizionario Biografico degli Italiani. Roma: Trecani (edición en línea: http://www.trecani.it/)

4 Regolamento dei Regi Spedali di Santa Maria Nuova e di Bonifazio. Firenze: Gaetano Cambiagi, 1789.

5 Regolamento dei Regi Spedali di Santa Maria Nouva e di Bonifazio. Firenze, Gaetano Cambiagi, 1789.

6 Tomado de la carta abierta de Carlo Levi (1823-1876) dirigida a Alexandre Brierre de Boismont (1797-1881) y publicada en el periódico La Nazione el 28 de abril de 1865, la traducción aquí utilizada corresponde a Huertas y Del Cura (2004), p. 124

7 En 1867 se publicó la 2ํo edición, cuya traducción al castellano es la que se ha consultado (Griesinger, 2007)

8 Todas las citas de la obra de Chiarugi están enumeradas según el parágrafo de la edición original, (Chiarugi, V. Della Pazzia in genere, e in specie. Trattato médico-analitico con una centuria di osservazioni, Firenze: Luigi Carlieri, Tomo I, 1793/ Tomo II y III, 1794), la traducción utilizada corresponde a la edición en castellano de Editorial Polemos, 2014.

9 “Un hombre que tiene la íntima convicción de una sensación que percibe actualmente cuando ningún objeto exterior que excite esta sensación llega a sus sentidos, se encuentra en estado de alucinación: es un visionario." (Esquirol, 1838 T. I, p.80).
10 La definición de melancolía y manía en función del tipo de delirio (parcial o general), que también será adoptado por Pinel (Pinel (1801) Tratado médico-filosófico sobre la enajenación mental o manía), corresponde a Thomas Willis (16211675), quien afirma que el delirio puede ser: "universal ... (en que) los perturbados tienen delirios con todas las cosas, o por lo menos la mayoría; hasta el punto de que no juzgan con clarividencia casi ningún tema ... (y otro) particular ... imaginan erróneamente en uno o dos casos determinados, pero en la mayor parte del resto de las cosas sus ideas no son muy incongruentes". Willis, T., (1683) Two Discourses Concerning the Soul of wich is that of the Vital and Sensitive of Man, Londres, pp. 188, (citado en Jackson, 1983).

11 En los siguientes autores encontramos claras referencias a la relación alternante entre manía y melancolía: Thomas WiIlis (1621-1675), Friedrich Hoffmann (1660-1742), Herman Boerhaave (1668-1738), Robert James (1705-1776), Giovanni Battista Morgagni (1728-1771), Willian Cullen (17101790), Joseph Townsend (1739-1816). Una aproximación a los textos y fragmentos en cuestión se podrá encontrar en Conti, 2006, pp. 27-29.

12 Siguiendo la propuesta epistemológica de George LantériLaura (2000) se puede aceptar que la mirada diacrónica no ingresa en el desarrollo de la clínica psiquiátrica hasta la segunda mitad del siglo XIX y solo en ese contexto puede emerger el concepto de entidad clínico evolutiva que será, a su entender, el núcleo duro del "paradigma de las enfermedades mentales" (1854-1926).

13 “... basar las distinciones de las enfermedades mentales en un conjunto de características subordinadas entre sí, y en su devenir, es decir, en la evolución de estos fenómenos que se suceden en un orden determinado ..." Falret, 1854. p. 382).

14 “... el diagnóstico [...] me hizo posible reconstruir el curso previo de la enfermedad a partir del estado actual del paciente; más aún, pude predecir el futuro desarrollo [...] en los detalles referentes a las diversas fases del cuadro sintomático..." (Kahlbaum, 1874). 


\section{BIBLIOGRAFÍA}

Berti Bock, G. (1971), "Ancora su Vincenzo Chiarugi: revisione bibliografica e breve analisi critica del suo pensiero", Acta Medica et Historica Patovina, 18, p 17.

Cabras, P., E. Campanilli y D. Lippi (1993), Uno psichiatra prima della psichiatria: Vincenzo Chiarugi ed il Trattato "Della pazzia in genere, e in specie" (1793-1794). Scientific Press, Florence.

Charland, L. (2014), “The distinction between 'Passion' and 'Emotion'. Vincenzo Chiarugi: a case study", History of Psychiatry, 25 (4), pp. 477-484.

Chiarugi, V. (1793), Della pazzia in genere, e in specie. Trattato medico-analitico, con una centuria di osservazioni, Florence, Luigi Carlieri.

Chiarugi, V. (2014), La Locura, sus géneros y especies, tratado médico-analítico con cien observaciones. Buenos Aires, Polemos.

Conti, N.A. (2008), “De la Alienación a las Enfermedades Mentales en Alemania: Karl Kahlbaum, un pensamiento de cambio", Vertex, Revista Argentina de Psiquiatría, vol. XIX (80), pp. 157-160.

Conti, N.A. (2006), "Trastorno Bipolar: desarrollo histórico y conceptual”. En: Akiskal A., M. Cetkovich-Bakmas; G. García Boneto; S. Strejilevich y G. Vázquez (comp.), Trastornos Bipolares, Buenos Aires, Panamericana, pp. 15-44.

Esquirol, J-ED (1838), Des maladies mentales considérées sous les rapports médical, hygiénique et médico-legal, 2 vol. Paris, Baillère.

Falret, J.P. (1854), "De la folie circulaire ou forme de maladie mentale caracterisée par l'alternative regulière de la manie et de la mélancolie", Bulletin de l'Academie de Medecine, $\mathrm{t}$. XIX, p. 382, Séance du 14 février.

Gerard, D.L. (1998) “Chiarugi and Pinel considered: Soul's brain/person's mind", Journal of the History of the Behavioral Sciences, 33 (4), pp. 381-403.

Grange, K.M. (1963), "Pinel or Chiarugi?”, Medical History, 7, pp. $371-380$

Griesinger, W. (2007) Patología y Terapéutica de las enfermedades mentales, Buenos Aires: Polemos.

Guarnieri, P. (1991), La storia Della psichiatria. Un secolo di studi in Italia. Firenze, Olschki.

Huertas, Rafael (2005), El siglo de la Clínica. Para una teoría de la práctica psiquiátrica. Madrid, Frenia.

Huertas, Rafael (1999), "Nosografía y Antinosografía en la Psiquiatría del Siglo XIX: en torno a la psicosis única", Revista Asociación Neuropsiquiátrica, 19 (69), pp. 63-76.
Huertas, Rafael y Mercedes del Cura (2004), "Chiarugi vs Pinel, la carta abierta de Carlo Livi a Brierre de Boismont", Frenia, 4 (2), pp. 109-133.

Jackson S (1983), "Melancholia and Partial Insaniti", J. Hist. Behav. Sci, 19, pp. 173-184.

Kahlbaum K (1874), Die Katatonie oder das Spannungsirresein, Berlín, Hirschwald.

Lantéri-Laura, G. (2000), Ensayo sobre los paradigmas de la psiquiatría moderna, Madrid, Triacastella.

Lantéri-Laura, G. (1994), “L'oeuvre de V. Chiarugi et la notion d'alienation mentale". En: Garrabé. J. (ed.), Philippe Pinel, Paris, Les empecheurs de penser en rond.

Lippi D. (2008), Medicina, chirurgia e sanità in Toscana tra'700 e '800: gli archivi inediti di Pietro Betti, Carlo Burci e Vincenzo Chiarugi. Firenze, Firenze University Press.

Lippi D. (1992), Nel privato di una grande vita: L'epistolario di Vincenzo Chiarugi. Roma, Vecchiarelli.

Maggini, Carlo y Antonello Pintus (2002), "Vicenzo Chiarugi (1759-1820)". En: Mario Maj y Filippo M. Ferro (eds.), Anthology of Italian Psychistric Texts, Paris, WPA.

Mora, G. (1987), Ed. and Trans. Vincenzo Chiarugi. On insanity and its classification. Canton, Mass.: Science History Publications.

Mora, G. (1959a), "Vincenzo Chiarugi (1759-1820) and his psychiatric reform in Florence in the late 18th century (on the occasion of the bi-centenary of his birth)", Journal of the History of Medicine and Allied Sciences, 14, pp. 424-33.

Mora, G. (1959b), "Bi-Centenary of the birth of Vincenzo Chiarugi (1749-1820) A Pioneer of the Modern Mental Hospital treatment", American Journal of Psychiatry, 116 (3), pp. 267-271.

Mora, G. (1954), “Vincenzo Chiarugi (1759-1820): His contribution to psychiatry", Bulletin of the Isaac Ray Medical Library, 2 (2), pp. 51-104.

Morel, P. (1996), Dictionnaire biographique de la psychiatrie. París, Les empecheurs de penser en rond.

Pallanti, S. (1996), "Vincenzo Chiarugi, 1759-1820", The American Journal of Psychiatry, 153 (7), p. 944.

Postel, J. y Quetel C. (2000), Nueva Historia de la Psiquiatría. Madrid, FCE.

Riefolo, G. (1991), "Uno spazio per la follia", Introducción a V. Chiarugi, Della Pazzia in genere, e in specie. Rome, Vecchiarelli. 
Scapini, A. (1966), La pazzia nell' interpretazione di Vincenzo Chiarugi. Pisa, Giardino.

Shorter, E. (2005), "Vincenzo Chiarugi". En: A Historical Dictionary of Psychiatry, Oxford. New York, Oxford University Press, p. 53.

Stagnaro, JC. (2014), "Estudio Preliminar". En: Chiarugi, V., La Locura, sus géneros y especies. tratado médico-analítico con cien observaciones. Buenos Aires: Polemos, pp. 9-80.
Stroppiana, L. (1976), "La reform degli ospedali psichiatrici di Chiarugi nel quadro del riformismo Toscano ed Europea" Rivista di storia della medicina, 20, pp: 168-179.

Tombaccini, D.; D. Lippi; F. Lelli y C. Rossi (2008), Florence and its Hospitals: A History of Health Care and Assistance in the Florentine Area. Firenze, Firenze University Press.

Zandri A., C. Acidini Luchinat y S. Francolini (1989), Lo spedale di messer Bonifazio. Roma, Mondadori Education. 